\title{
Übersicht
}

Obere Extremität 2021 $\cdot 16: 215-220$

https://doi.org/10.1007/s11678-020-00615-8

Eingegangen: 14. Juli 2020

Angenommen: 4. November 2020

Online publiziert: 4. Dezember 2020

(c) Der/die Autor(en) 2020

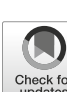

\author{
Malik Jessen' $\cdot$ Sebastian Lappen ${ }^{2}$ (D) $\cdot$ Marco Schneider $^{3,4}$ \\ ${ }^{1}$ Medizinische Fakultät Mannheim, Ruprecht-Karls-Universität Heidelberg, Mannheim, Deutschland \\ ${ }^{2}$ Abteilung und Poliklinik für Sportorthopädie des Klinikums rechts der Isar, Technische Universität \\ München, München, Deutschland \\ ${ }^{3}$ Klinik für Unfall-, Handchirurgie und Sportmedizin, St. Vincentius-Kliniken, Karlsruhe, Deutschland \\ ${ }^{4}$ Universität Witten/Herdecke, Witten, Deutschland
}

\section{„Patient-reported outcome measures" (PROMs) in der Schulter- und Ellenbogenchirurgie}

\begin{abstract}
„Patient-reported outcome measures" (PROMs) erlauben die Sicht auf den subjektiv wahrgenommenen Gesundheitsstatus von Patienten. Es wird eine Vielzahl an unterschiedlichen Schulter- und EllenbogenPROMs beschrieben, welche jedoch uneinheitlich genutzt werden. $\mathrm{Zu}$ dem fehlen für viele dieser Scores validierte deutsche Übersetzungen.
\end{abstract}

In den letzten Jahrzehnten hat die subjektive Sicht der Patienten auf ihren eigenen Gesundheitsstatus zunehmend an Bedeutung gewonnen [3]. PROMs messen diesen subjektiven Gesundheitsstatus und den selbst erlebten Einfluss von Pathologien auf Gesundheit und Funktionalität in Form von standardisierten, validierten Fragebögen [10, 11, 23, 42]. Es werden generische, gelenkspezifische und krankheitsspezifische PROMs unterschieden. Generische PROMs wie der European Quality of Life 5 Dimensions (EQ5D), Short Form 36 (SF-36) oder Veterans Rand 12 (VR-12) messen unterschiedliche Aspekte einer Vielzahl von Erkrankungen und ermöglichen so die Gesamtbewertung von Versorgung, Lebensqualität und Kosteneffizienz von Interventionen [28]. Sie bestimmen die allgemeine gesundheitsbezogene Lebensqualität und können krankheitsunabhängig ein-

Die Autoren M. Jessen und S. Lappen haben zu gleichen Teilen zum Manuskript beigetragen. gesetzt werden [23]. Krankheitsspezifische PROMs messen den Schweregrad einer spezifischen Erkrankung oder bestimmte Aspekte einer Pathologie, welche in den meisten Fällen durch ein generisches Instrument nicht ausreichend erfasst werden können [11,23]. Generische und krankheitsspezifische PROMs werden in der Regel kombiniert verwendet [28].

Obwohl die Großzahl von PROMs für Forschungszwecke entwickelt wurde, konnten auch positive Effekte der Erhebung von PROMs im klinischen Alltag nachgewiesen werden. So zeigte sich ein Zusammenhang zwischen der routinemäßigen Erfassung von PROMs mit einer verbesserten Arzt-Patienten-Kommunikation und einer erhöhten Patientencompliance $[7,20,23,38]$. Auch gesundheitsökonomisch gewinnen PROMs mit dem internationalen Trend zur routinemäßigen Erfassung als Grundlage für die ärztliche Abrechnung zunehmend an Bedeutung $[4,7,23]$.

Bei der Verwendung von PROMs können jedoch zahlreiche technische, soziale, kulturelle, rechtliche und logistische Hindernisse auftreten [28, 38]. Hinzu kommt eine international häufig unterschiedliche Handhabung und Auswahl von PROMs [5]. Auch ist zu beachten, dass die meisten PROMs im englischsprachigen Raum und für angelsächsische Kulturen entwickelt wurden [17]. Um das Risiko einer Verzerrung oder einer erhöhten Variabilität zu vermeiden, müssen angemessene Übersetzungen mit transkulturellen Anpassungen und anschließender Validierung nach definierten Vorgaben vorgenommen werden [13].

\section{PROMs in der Schulterchirurgie}

In der Schulterchirurgie spielen PROMs eine wichtige Rolle. Es werden über 30 verschiedene Schulterscores beschrieben, von denen viele jedoch ohne entsprechende statistische Validierung erstellt und aus weitgehend historischen Gründen verwendet werden [49].

Die weltweit am häufigsten genutzten PROMs in Studien zu Schulterpathologien sind laut einer Analyse von Mosher et al. [37] der Constant-Murley Score (CS), der American Shoulder and Elbow Surgeons Shoulder Form (ASES), die Visual Analog Scale (VAS), der Simple Shoulder Test (SST) und die University of California, Los Angeles, Shoulder Rating Scale (UCLA). Laut einer Analyse von Booker et al. [37] bestehen jedoch Unterschiede in der internationalen Nutzung von Schulter-PROMs. Insbesondere der Oxford Shoulder Score (OSS) wird im europäischen Raum häufiger genutzt als in der amerikanischen Literatur [37].

Zwar gibt es für viele der international geläufigen PROMs validierte, deutsche Übersetzungen, eine Analyse zur Nutzung im deutschsprachigen Raum exis- 


\begin{tabular}{|c|c|c|c|c|}
\hline Score & „Patient-reported" & "Clinician-reported" & $\begin{array}{l}\text { Maximale } \\
\text { Punktzahl }\end{array}$ & $\begin{array}{l}\text { Validierte deutsche } \\
\text { Übersetzung }\end{array}$ \\
\hline ASES & $\begin{array}{l}\text { Schmerz, } \\
\text { Instabilität, } \\
\text { ADL }\end{array}$ & $\begin{array}{l}\text { ROM, } \\
\text { Kraft, } \\
\text { Instabilität, } \\
\text { klinische Untersu- } \\
\text { chungszeichen }\end{array}$ & 100 von 100 & $\begin{array}{l}\text { Goldhahn J. et al., } \\
2008 \text { [16] }\end{array}$ \\
\hline$C S$ & $\begin{array}{l}\text { Schmerz, } \\
\text { ADL }\end{array}$ & $\begin{array}{l}\text { ROM, } \\
\text { Kraft }\end{array}$ & 100 von 100 & Constant CR, 1991 [8] \\
\hline$D A S H$ & $\begin{array}{l}\text { Funktionalität, } \\
\text { Schmerz, } \\
\text { Schlafstörungen }\end{array}$ & - & 0 von 100 & $\begin{array}{l}\text { DASH: Germann G } \\
\text { et al., } 2003[15,32] \\
\text { Quick-DASH: - }\end{array}$ \\
\hline OSS & $\begin{array}{l}\text { Schmerz, } \\
\text { Funktionalität }\end{array}$ & - & 12 von 60 & Huber et al., 2004 [24] \\
\hline $\begin{array}{l}\text { Rowe } \\
\text { Score }\end{array}$ & $\begin{array}{l}\text { Instabilität, } \\
\text { Funktionalität, } \\
\text { ROM }\end{array}$ & - & 100 von 100 & $\begin{array}{l}\text { Jensen KU et al., } 2009 \\
\text { [25] }\end{array}$ \\
\hline SST & $\begin{array}{l}\text { Funktionalität, } \\
\text { Schmerzen, } \\
\text { Kraft, } \\
\text { Schlafstörungen }\end{array}$ & - & 12 von 12 & - \\
\hline $\begin{array}{l}\text { SSV } \\
\text { SANE } \\
\text { SPONSA }\end{array}$ & Krankheitswert & - & 100 von 100 & - \\
\hline$U C L A$ & $\begin{array}{l}\text { Schmerz, } \\
\text { Funktionalität, } \\
\text { Kraft, } \\
\text { ROM (Zufriedenheit) }\end{array}$ & - & 30 & - \\
\hline WOSI & $\begin{array}{l}\text { Symptome } \\
\text { Sport, Freizeit und } \\
\text { Arbeit, } \\
\text { ADL, } \\
\text { emotionale Funktion }\end{array}$ & - & 0 von 2100 & $\begin{array}{l}\text { Hofstaetter et al., } \\
2010 \text { [22] }\end{array}$ \\
\hline \multicolumn{5}{|c|}{$\begin{array}{l}\text { UCLA University of California, Los Angeles, Shoulder Rating Scale, OSS Oxford Shoulder Score, } \\
\text { CS Constant-Murley Score, ASES American Shoulder and Elbow Surgeons Shoulder Form, SST } \\
\text { Simple Shoulder Test, DASH Disability of Shoulder, Arms and Hand Questionnaire, WOSI Western } \\
\text { Ontario Shoulder Instability Index, SSV Subjective Shoulder Value, SANE Single Assessment Numeric } \\
\text { Evaluation Score, SPONSA Stanmore Percentage of Normal Shoulder Assessment, ROM "range of } \\
\text { motion", ADL "activities of daily living" }\end{array}$} \\
\hline
\end{tabular}

tiert zum jetzigen Zeitpunkt jedoch nicht $[8,15,16,24,25,32]$.

Die - Tab. 1 zeigt die Auflistung der geläufigsten Schulterscores.

\section{American Shoulder and Elbow Surgeons Shoulder Form (ASES)}

Der 1994 entwickelte Score besteht aus einem 11 Fragen umfassenden Patientenfragebogen („patient self-assessment“, pASES) und einem optionalen klinischen Evaluationsbogen („clinical assessment", cASES), welcher nicht in die Berechnung der Punktzahl einfließt [18]. Der ASES ist somit ein PROM, der durch die Sicht des Klinikers ergänzt werden kann. Er ist der weltweit am zweithäufigsten angewand-

\section{Constant-Murley Score (CS)}

Der CS wurde 1987 erstmalig publiziert und besteht aus einem vom Patienten und einem vom Untersucher erhobenen Anteil und ist somit kein reiner PROM [16, 32]. Mit $35 \mathrm{zu} 65$ Punkten machen die vom Patienten erfassten Parameter den kleineren Anteil aus. Der CS ist der weltweit am häufigsten verwendete Schulterscore und wird führend bei Studien zu arthroskopischen Eingriffen wie Rotatorenmanschettenrekonstruktionen, bei glenohumeralen Stabilisierungen und bei Humeruskopffrakturen verwendet [37]. Er ist leicht anzuwenden und aufgrund seiner langfristigen Verwendung ermöglicht er den Vergleich von älteren in der Literatur berichteten Behandlungsergebnisse mit moderneren chirurgischen oder nichtchirurgischen Therapien [37]. Der CS wurde aufgrund mangelhafter Standardisierung (v.a. die Messung der Abduktionskraft) sowie dem fehlenden Erfassen der Schulterinstabilität kritisiert $[16,32]$. Weitere Kritik wurde aufgrund von unterschiedlichen Ergebnissen für Männer und Frauen und der Verringerung der Punktzahlen mit zunehmendem Alter geäußert [37], sodass eine normierte alters- und geschlechtsgewichtete Version entwickelt wurde [37].

Eine erstmalige deutsche Übersetzung erfolgte durch Constant 1991 [8,32], 2003 erfolgte die Normierung für die Deutsche und 2005 die Normierung für die Schweizer Bevölkerung [8, 32].

\section{Disabilities of the Arm, Shoulder and Hand (DASH) und Quick-DASH (qDASH)}

Der DASH-Fragebogen erfasst alle Gelenke der oberen Extremität [37]. Er besteht aus einem 30 Fragen umfassenden obligatorischen Modul und den je 4 Fragen umfassenden optionalen Arbeits- und Berufsmodul sowie Sportund Musikmodul. Der qDASH ist eine aus 11 Fragen bestehende Kurzversion des DASH, welcher analog erhoben wird [37]. Der DASH ist der in Studien zu Pathologien der Klavikula am häufigsten genutzte Score [37] und zeigt eine gute Validität und Reliabilität [5]. 2003 publizierten Germann et al. [15] eine 
validierte deutsche Übersetzung, für den qDASH existiert aktuell keine validierte deutsche Form [32].

\section{Oxford Shoulder Score (OSS)}

Der OSS besteht aus 12 Fragen und wurde zur postoperativen Evaluation entwickelt $[24,32]$. Er wird insbesondere im europäischen Raum verwendet [37]. Da der OSS die Schulterinstabilität nicht miterfasst, wurde ergänzend der Oxford Shoulder Instability Score (OSIS) entwickelt [24, 32].

Der OSS stellt ein robustes Instrument zur postoperativen Erhebung von „patient-reported outcome“ (PRO) dar, weitere Untersuchung bezüglich des Einflusses von Faktoren wie Alter und Geschlecht auf den OSS sollten jedoch durchgeführt werden [24, 32]. Eine validierte deutsche Version wurde 2004 von Huber et al. [24] publiziert [32].

\section{Rowe Shoulder Score}

Der Rowe Score wurde 1978 erstmalig als Instrument zur Evaluation von postoperativen Ergebnissen nach BankartRepair beschrieben [44]. Er ist der weltweit am häufigsten genutzte Score für Studien zur Schulterinstabilität, existiert jedoch in verschiedenen Versionen, die parallel und ohne exakter Angabe der Version verwendet werden $[43,45,46]$. Zwischen den verschiedenen Rowe-ScoreVersionen wurden signifikante Abweichungen der erreichten Punktzahl und Unterschiede der Bewertungsergebnisse nachgewiesen [44]. 2009 wurde von Jensen et al. [25] ein deutscher Selbsteinschätzungsbogen auf Grundlage des Rowe-Scores publiziert [32].

\section{Simple Shoulder Test (SST)}

Der 1993 veröffentlichte SST besteht aus 12 Fragen, die dichotom mittels „Ja“ oder „Nein“ beantwortet werden können [30]. Er ist der weltweit am vierthäufigsten genutzte Score in Schulterstudien. Der SST ist valide, reliabel und gilt als benutzerfreundlich [25, 32]. Er wird insbesondere in Nordamerika häufig genutzt [25, 32]. Bemängelt wird am SST, dass zum einen hohe Werte auch beim Vorliegen

Obere Extremität 2021 · 16:215-220 https://doi.org/10.1007/s11678-020-00615-8

(c) Der/die Autor(en) 2020

\section{„Patient-reported outcome measures" (PROMs) in der Schulter- und Ellenbogenchirurgie}

Zusammenfassung

„Patient-reported outcome measures" (PROMs) sind Instrumente, mit denen der subjektive Gesundheitszustand eines Patienten gemessen werden kann. Sie gewinnen zunehmend an wissenschaftlicher, klinischer und gesundheitsökonomischer Bedeutung. Man unterscheidet zwischen generischen, gelenkspezifischen und krankheitsspezifischen PROMs. Um das Risiko von Verzerrungen oder erhöhter Variabilität zu vermeiden, müssen geeignete Übersetzungen mit transkulturellen Anpassungen und anschließender Validierung gemäß definierten Richtlinien durchgeführt werden. Für viele gängige Schulter- und Ellenbogenwerte existiert bis dato noch keine validierte Übersetzung. Die weltweit am häufigsten verwendeten Schulter-PROMs sind der Constant-Murley Score, der American Shoulder and Elbow Surgeons Shoulder Form und der Simple Shoulder Test. Studien zu Ellenbogenpathologien verwenden am häufigsten den Mayo Elbow Performance Score und den Disabilities of the Arm, Shoulder and Hand Score. Derzeit besteht jedoch kein wissenschaftlicher Konsens über die Verwendung von Schulter- und Ellenbogen-PROMs.

Schlüsselwörter Ergebnisbewertung · Score · Evaluierung . Gesundheitsstatus · Lebensqualität

\section{Patient-reported outcome measures (PROMs) in shoulder and elbow surgery}

\section{Abstract}

Patient-reported outcome measures (PROMs) are instruments that can be used to measure a patient's subjective health status. They are gaining increasing scientific, clinical, and health economic importance. A distinction is made between generic, joint-specific, and disease-specific PROMs. In order to avoid the risk of bias or increased variability, appropriate translations with transcultural adjustments and subsequent validation must be carried out according to defined guidelines. However, there are no corresponding translations for many common shoulder and elbow scores. The most widely used shoulder PROMs worldwide are the Constant-Murley Score, the American Shoulder and Elbow Surgeons Shoulder Form, and the Simple Shoulder Test. Studies on elbow pathologies most often use the Mayo Elbow Performance Score and the Disabilities of the Arm, Shoulder and Hand Score. However, there is currently no scientific consensus on the use of shoulder and elbow scores.

\section{Keywords}

Outcome assessment . Scoring methods . Evaluation · Health status · Quality of life einer signifikanten Pathologie auftreten können und zum anderen Faktoren wie Alter oder Art der Pathologie das Scoreergebnis uneinheitlich beeinflussen. Eine deutsche Übersetzung (gemeinsam mit dem „shoulder activity level“) ist bereits erfolgt und wird nach der Validierungsphase von den Autoren dieses Artikels veröffentlicht.

\section{Single Question Subjective Measures}

Zu den Single Question Subjective Measures zählen der Subjective Shoulder Va- lue (SSV; $[25,32]$ ), der Single Assessment Numeric Evaluation (SANE; [25, 32]) und das Stanmore Percentage of Normal Shoulder Assessment (SPONSA; [25, 32]). In allen drei Scores bewerten die $\mathrm{Pa}$ tienten in einer einzelnen Frage den aktuellen Krankheitswert ihrer Schulter; der SANE lässt sich jedoch auch auf andere Gelenke anwenden. Insbesondere für den SANE konnte eine gute Korrelation mit anderen Schulter-PROMs nachgewiesen werden [19, 39]. 


\section{Übersicht}

\begin{tabular}{|c|c|c|c|c|}
\hline Score & „Patient-reported" & "Clinician-reported" & $\begin{array}{l}\text { Maximale } \\
\text { Punktzahl }\end{array}$ & $\begin{array}{l}\text { Validierte deutsche } \\
\text { Übersetzung }\end{array}$ \\
\hline ASES-e & $\begin{array}{l}\text { Schmerz, } \\
\text { ADL, } \\
\text { Zufriedenheit }\end{array}$ & $\begin{array}{l}\text { ROM, } \\
\text { Kraft, } \\
\text { Instabilität, } \\
\text { klinische Untersu- } \\
\text { chungszeichen }\end{array}$ & 100 von 100 & $\begin{array}{l}\text { John M. et al., } 2010 \\
\text { [26] }\end{array}$ \\
\hline DASH & $\begin{array}{l}\text { Funktionalität, } \\
\text { Schmerz, } \\
\text { Schlafstörungen }\end{array}$ & - & 0 von 100 & $\begin{array}{l}\text { DASH: Germann G } \\
\text { et al., } 2003[15,32] \\
\text { Quick-DASH: - }\end{array}$ \\
\hline MEPS & $\begin{array}{l}\text { Schmerz, } \\
\text { ADL }\end{array}$ & $\begin{array}{l}\text { ROM, } \\
\text { Instabilität }\end{array}$ & 100 von 100 & $\begin{array}{l}\text { Schneider MM et al., } \\
2019 \text { [47] }\end{array}$ \\
\hline OES & $\begin{array}{l}\text { Schmerz, } \\
\text { Funktionalität, } \\
\text { sozialpsychologische } \\
\text { Aspekte }\end{array}$ & - & 100 von 100 & $\begin{array}{l}\text { Marquardt et al., } 2016 \\
\text { [34] }\end{array}$ \\
\hline PRTEE & $\begin{array}{l}\text { Schmerz, } \\
\text { Funktionalität, } \\
\text { ADL }\end{array}$ & - & 0 von 100 & $\begin{array}{l}\text { Marks M. et al., } 2020 \\
\text { [33] }\end{array}$ \\
\hline \multicolumn{5}{|c|}{$\begin{array}{l}\text { MEPS Mayo Elbow Performance Score, DASH Disability of Shoulder, Arms and Hand Questionnaire, } \\
\text { PRTEE Patient-Rated Tennis Elbow Evaluation, OES Oxford Elbow Score, ASES-e American Shoulder } \\
\text { and Elbow Surgeons Shoulder Form-elbow, ROM "range of motion", } A D L_{\text {"activities of daily living" }}\end{array}$} \\
\hline
\end{tabular}

University of California at Los Angeles (UCLA) Shoulder-RatingSystem

Der UCLA wurde 1981 zur Beurteilung von Patienten nach Schulterendoprothetik veröffentlicht [1], wird jedoch auch bei Patienten mit anderen Schultererkrankungen angewendet [1]. Nach der Modifizierung von Ellman et al. [12] wurde 1986 die Kategorie Muskelkraft und Beweglichkeit in aktive Flexion und Flexionskraft unterteilt sowie der Parameter Patientenzufriedenheit aufgenommen. Die maximale Punktzahl wurde durch diese Veränderung von 30 auf 35 Punkte erhöht. Aufgrund des Parameters „Patientenzufriedenheit“ lässt sich der Score jedoch nur postoperativ erheben. Zudem ist nicht eindeutig definiert, wie ein unveränderter Zustand postoperativ zu klassifizieren ist, was eine geringe Reliabilität, Validität und Empfindlichkeit bedingen kann [1]. Eine validierte deutsche Übersetzung existiert aktuell nicht.

\section{Western Ontario Shoulder Instability Index (WOSI)}

Der 1998 veröffentlichte WOSI ist ein Schulterinstabilitätsscore [29]. Er ist valide, reliabel und zeigt eine bessere
Empfindlichkeit für Instabilitäten verglichen mit anderen geläufigen Schulterscores [14]. Eine validierte deutsche Übersetzung wurde 2010 publiziert [22].

\section{Ellenbogen-PROMs}

Die Verwendung von klinischen Outcomescores zur Evaluation des Ellenbogens nimmt seit Anfang der 1990er-Jahre annähernd exponentiell zu [14]. Bis zum Jahr 2016 wurden in der Literatur 72 unterschiedliche Ellenbogenoutcomescores beschrieben [14].

Obwohl regionsabhängig einzelne Unterschiede in deren Verwendung nachgewiesen werden konnten, stellen unabhängig der Lokalisation der Mayo Elbow Performance Score (MEPS) und der DASH weltweit die meistverwendeten Ellenbogenscores dar [14].

Die - Tab. 2 zeigt eine Zusammenfassung der wichtigsten Scores für den Ellenbogen.

\section{American Shoulder and Elbow Surgeons - elbow (ASES-e)}

Der 1999 veröffentlichte ASES-e [27] wird hauptsächlich in der nordamerikanischen und weniger in der europäischen Literatur verwendet [14]. Er findet insbesondere Anwendung bei traumatisch be- dingten Pathologien. Analog zum ASES gliedert sich der ASES-e in einen Anteil der Patientenselbstevaluation (,patient self-evaluation", pASES-e) und einen Untersucheranteil (,clinician part“, cASESe), welche getrennt betrachtet werden [27]. Der ASES-e ist somit ebenfalls kein reiner PROM sondern fasst ebenfalls die Sicht des Klinkers mit ein. Er hat sich als valides, reliables und empfindliches Instrument mit guter Korrelationen $\mathrm{zu}$ weiteren Scores erwiesen [27]. Seit 2010 verfügt der pASES-e über eine validierte, deutsche Übersetzung [26].

\section{Mayo Elbow Performance Score (MEPS)}

Der von Morrey und Adams 1992 beschriebene MEPS [36] stellt den meistverwendeten Score zur Evaluation des Ellenbogens in der Literatur mit stets zunehmender Bedeutung dar [14, 41], obwohl dieser nicht mit einer formalen Methodik entwickelt wurde und der Untersucheranteil des Scores uneinheitlich angewendet wird [41]. Der Score besteht aus 12 Fragen, von denen 6 Fragen vom Patienten und 6 Frage vom Untersucher beantwortet werden, sodass es sich beim MEPS um keinen reinen PROM handelt. Es existieren verschiedene Versionen des Scores $[6,14]$, welche aktuell jedoch nicht mehr genutzt werden und sich in erster Linie durch die unterschiedlich formulierte Frage zum Schmerz unterscheiden. Seit 2019 gibt es eine validierte deutsche Übersetzung der inzwischen gängigsten Version [47].

\section{Oxford Elbow Score (OES)}

Der 2009 [9] veröffentlichte OES besteht aus 12 Fragen, die ausschließlich von Patienten beantwortet werden. Der OES ist ein verlässliches Instrument zur Bewertung der Lebensqualität im Zusammenhang mit Ellenbogenpathologien [9]. Allerdings wird von der Oxford University Innovation eine Gebühr für die Nutzung des OES bei industriefinanzierte Studien und kommerziellen Gebrauch verlangt [2]. Seit 2016 existiert eine validierte deutsche Version des OES [34, 35]. 
Tab. 3 Scoreempfehlungen des Executive Committee der American Shoulder and Elbow Surgeons Shoulder Form (ASES, [21])

\begin{tabular}{|c|c|c|}
\hline & Schulter & Ellenbogen \\
\hline Generischer Score & \multicolumn{2}{|l|}{ VR-12 } \\
\hline \multirow[t]{2}{*}{ Gelenkspezifischer Score } & \multicolumn{2}{|l|}{ SANE } \\
\hline & ASES-Score & Quick-DASH \\
\hline $\begin{array}{l}\text { Ergänzung für wissenschaftliche } \\
\text { Zwecke }\end{array}$ & $\begin{array}{l}\text { Pathologiespezifisch (WORC, WOSI oder } \\
\text { WOOS), } \\
\text { PENN }\end{array}$ & MEPS \\
\hline \multicolumn{3}{|c|}{$\begin{array}{l}\text { PENN Pennsylvania Shoulder Score, VR-12 Veteran's Rand 12, SANE Single Assessment Numeric } \\
\text { Evaluation Score, ASES American Shoulder and Elbow Surgeons Shoulder Form, DASH Disability } \\
\text { of Shoulder, Arms and Hand Questionnaire, MEPS Mayo Elbow Performance Score, WOSI Western } \\
\text { Ontario Shoulder Instability Index, WORC Western Ontario Rotator Cuff, WOOS Western Ontario } \\
\text { Osteoarthritis Shoulder }\end{array}$} \\
\hline
\end{tabular}

\section{Patient-Rated Tennis Elbow Evaluation (PRTEE)}

Der 15 Fragen umfassende PRTEE [40] stellt den meistverwendeten Ellenbogenscore bei Tendinopathien des Ellenbogens dar [14]. Der PRTEE weist eine gute Validität, Reliabilität und Korrelation mit anderen geläufigen Ellenbogenscores auf [27]. Glanzmann et al. [33] haben kürzlich eine validierte Übersetzung bereitgestellt.

\section{Anwendung}

Aufgrund der Vielzahl an unterschiedlichen Schulter- und Ellenbogenscores sowie deren unterschiedlicher Handhabung kann die Auswahl eines geeigneten Messinstruments schwierig sein. Bestrebungen zur Vereinfachung der Auswahl von Scores wurden mit der Definition von "core outcome sets" (COS), einem vereinbarten Minimum an Outcomeparametern, die in allen Studien einer bestimmten Pathologie genutzt werden sollen, unternommen [31]. COS werden auf Grundlage einer Konsensentscheidung von Experten gebildet und für definierte Vorhaben empfohlen [48]. Sie dienen der Schaffung einer verbesserten Vergleichbarkeit und sollen zur Verbesserung der Evidenz verschiedener Therapien im internationalen Vergleich beitragen. Empfehlungen zur Scoreauswahl in der Schulter- und Ellenbogenchirurgie wurden durch das Executive Commitee der ASES ausgesprochen (- Tab. 3; [21]). Hier wurden validierte PROMs mit guter Psychometrie und größtmöglicher Benutzerfreundlichkeit für Patienten und
Ärzte gesucht. Allerdings wurden die USBevölkerungsnormen in erster Linie berücksichtigt, sodass der Veteran's Rand 12 (VR-12) als generischer Score in die Empfehlung aufgenommen wurde, da dieser Teil der Medicare Health Outcomes Survey ist. Der ASES-Score wurde ebenfalls empfohlen, obwohl er außerhalb der USA weniger relevant ist. Die Verwendung des OSS wird jedoch vom ASES-Komitee als Alternative zum ASES-Score akzeptiert, da er über gute psychometrische Daten verfügt und in Europa weit verbreitet ist.

Ein Konsens in der Literatur konnte bislang jedoch nicht erreicht werden, sodass insbesondere mit Blick auf die wachsende Bedeutung von PROMs weitere Bemühungen zum einheitlichen Gebrauch notwendig sind.

\section{Fazit für die Praxis}

- Die Verwendung von PROMs („patient-reported outcome measures") in der Schulter- und Ellenbogenchirurgie gewinnt zunehmend wissenschaftlich, klinisch und auch gesundheitsökonomisch an Bedeutung.

- PROMs müssen mit transkulturellen Anpassungen übersetzt und anschließend validiert werden, um das Risiko einer Verzerrung oder einer erhöhten Variabilität zu vermeiden.

- Es sollten generische, gelenkspezifische und pathologiespezifische Scores kombiniert genutzt werden.

- Zu den am häufigsten in der Schulterund Ellenbogenchirurgie genutzten Scores zählen der Disabilities of Arm, Shoulder and Hand (DASH) Score, der
Constant Score (CS), der American Shoulder and Elbow Score (ASES) und der Mayo Elbow Performance Score (MEPS). Für einige gängige PROMs existieren jedoch keine validierten deutschen Übersetzungen.

- Bisher bestehen kein internationaler Konsens und keine einheitliche Nutzung von PROMs in der Schulterund Ellenbogenchirurgie.

\begin{tabular}{l} 
Dorrespondenzadresse \\
$\begin{array}{l}\text { Abteilung und Poliklinik } \\
\text { für Sportorthopädie des } \\
\text { Klinikums rechts der Isar, } \\
\text { Technische Universität } \\
\text { München } \\
\text { Ismaninger Str. 22, } \\
\text { 81675 München, Deutschland } \\
\text { sebastian.lappen@web.de }\end{array}$ \\
\hline
\end{tabular}

Funding. Open Access funding enabled and organized by Projekt DEAL.

\section{Einhaltung ethischer Richtlinien}

Interessenkonflikt. M. Jessen, S. Lappen und M. Schneider geben an, dass kein Interessenkonflikt besteht.

Alle beschriebenen Untersuchungen am Menschen oder an menschlichem Gewebe wurden mit Zustimmung der zuständigen Ethikkommission, im Einklang mit nationalem Recht sowie gemäß der Deklaration von Helsinki von 1975 (in der aktuellen, überarbeiteten Fassung) durchgeführt. Von allen beteiligten Patienten liegt eine Einverständniserklärung vor.

Open Access. Dieser Artikel wird unter der Creative Commons Namensnennung 4.0 International Lizenz veröffentlicht, welche die Nutzung, Vervielfältigung, Bearbeitung, Verbreitung und Wiedergabe in jeglichem Medium und Format erlaubt, sofern Sie den/die ursprünglichen Autor(en) und die Quelle ordnungsgemäß nennen, einen Link zur Creative Commons Lizenz beifügen und angeben, ob Änderungen vorgenommen wurden.

Die in diesem Artikel enthaltenen Bilder und sonstiges Drittmaterial unterliegen ebenfalls der genannten Creative Commons Lizenz, sofern sich aus der Abbildungslegende nichts anderes ergibt. Sofern das betreffende Material nicht unter der genannten Creative Commons Lizenz steht und die betreffende Handlung nicht nach gesetzlichen Vorschriften erlaubt ist, ist für die oben aufgeführten Weiterverwendungen des Materials die Einwilligung des jeweiligen Rechteinhabers einzuholen.

Weitere Details zur Lizenz entnehmen Sie bitte der Lizenzinformation auf http://creativecommons.org/ licenses/by/4.0/deed.de. 


\section{Literatur}

1. Amstutz HC, Sew Hoy AL, Clarke IC (1981) UCLA anatomic total shoulder arthroplasty. Clin Orthop Relat Res 155:7-20

2. Oxford University Innovation Click-To-Licence Portal.https://process.innovation.ox.ac.uk/.Zugegriffen: 4. Juli 2020

3. Bayley KB, London MR, Grunkemeier GL et al (1995) Measuring the success of treatment in patient terms. Med Care 33:AS226-AS235

4. BlackN (2013) Patient reported outcome measures could help transform healthcare. BMJ 346:f167

5. Booker S, Alfahad N, Scott M et al (2015) Use of scoring systems for assessing and reporting the outcome results from shoulder surgery and arthroplasty. World JOrthop 6:244-251

6. Broberg MA, Morrey BF (1986) Results of delayed excision of the radial head after fracture. J Bone Joint Surg Am 68:669-674

7. Chen J, Ou L, Hollis SJ (2013) A systematic review of the impact of routine collection of patient reported outcome measures on patients, providers and health organisations in an oncologic setting. BMC Health Serv Res 13:211

8. Constant CR (1991) Schulterfunktionsbeurteilung. Orthopäde 20:289-294

9. Dawson J, Doll H, Boller I et al (2008) The development and validation of a patient-reported questionnaire to assess outcomes of elbow surgery. J Bone Joint Surg Br 90:466-473

10. Dawson J, Doll H, Fitzpatrick R et al (2010) The routine use of patient reported outcome measures in healthcare settings. BMJ340:C186

11. Devlin NJ, Appleby J (2010) Getting the most out of PROMS. Putting health outcomes at the heart of NHS decision-making. The King's Fund, London

12. Ellman H, Hanker G, Bayer M (1986) Repair of the rotator cuff. End-result study of factors influencing reconstruction. J Bone Joint Surg Am 68:1136-1144

13. Eremenco S, Pease S, Mann S et al (2017) PatientReported Outcome (PRO) Consortium translation process: consensus development of updated best practices. J Patient Rep Outcomes 2:12

14. Evans JP, Smith CD, Fine NF et al (2018) Clinical rating systems in elbow research - a systematic review exploring trends and distributions of use. JShoulder Elbow Surg 27:e98-e106

15. Germann G, Harth A, Wind G et al (2003) Standardisation and validation of the German version 2.0 of the Disability of Arm, Shoulder, Hand (DASH) questionnaire. Unfallchirurg 106:13-19

16. Goldhahn J, Angst F, Drerup S et al (2008) Lessons learned during the cross-cultural adaptation of the American Shoulder and Elbow Surgeons shoulder form into German. J Shoulder Elbow Surg 17:248-254

17. Gomez-ValeroS, Garcia-PerezF,Florez-Garcia MTet al (2017) Assessment of cross-cultural adaptations of patient-reported shoulder outcome measures in Spanish: a systematic review. Shoulder Elbow 9:233-246

18. Goodman J, Lau BC, Krupp RJ et al (2018) Clinical measurements versus patient-reported outcomes: analysis of the American Shoulder and Elbow Surgeons physician assessment in patients undergoing reverse total shoulder arthroplasty. JSES Open Access 2:144-149

19. Gowd AK, Charles MD, Liu JN et al (2019) Single Assessment Numeric Evaluation (SANE) is a reliable metric to measure clinically significant improvements following shoulder arthroplasty. JShoulder Elbow Surg 28:2238-2246
20. Griggs CL, Schneider JC, Kazis LE et al (2017) Patient-reported outcome measures: a stethoscope for the patient history. Ann Surg 265:1066-1067

21. Hawkins RJ, Thigpen CA (2018) Selection, implementation, and interpretation of patient-centered shoulder and elbow outcomes. J Shoulder Elbow Surg 27:357-362

22. Hofstaetter JG, Hanslik-Schnabel B, Hofstaetter SG et al (2010) Cross-cultural adaptation and validation of the German version of the Western Ontario Shoulder Instability index. Arch Orthop Trauma Surg 130:787-796

23. Hostettlera S, Kraftb E, Bosshardc C (2018) Patientreported outcome mea-sures: Die Patientensicht zählt. Schweiz Ärzteztg 99(40):1348-1352. https:// doi.org/10.4414/saez.2018.17187

24. Huber W, Hofstaetter JG, Hanslik-Schnabel B et al (2004) The German version of the Oxford Shoulder Score-cross-cultural adaptation and validation. Arch Orthop Trauma Surg 124:531-536

25. Jensen KU, Bongaerts G, Schneider S et al (2009) Selbsteinschätzungsbogen auf Grundlage des Rowe-Scores von 1982. Obere Extrem 4:178-183

26. John M, Angst F, Awiszus F et al (2010) The American Shoulder and Elbow Surgeons Elbow Questionnaire: cross-cultural adaptation into German and evaluation of its psychometric properties. JHand Ther 23:301-313 (quiz 314)

27. King GJ, Richards RR, Zuckerman JD et al (1999) A standardized method for assessment of elbow function. Research Committee, American Shoulder and Elbow Surgeons. J Shoulder Elbow Surg 8:351-354

28. Kingsley C, Patel S (2017) Patient-reported outcome measures and patient-reported experience measures. BJA Educ 17:137-144

29. Kirkley A, Griffin S, Mclintock $H$ et al (1998) The development and evaluation of a diseasespecific quality of life measurement tool for shoulder instability. The Western Ontario Shoulder Instability Index (WOSI). Am J Sports Med 26:764-772

30. Lippitt SB, Harryman DT, Matsen FA (1993) A practical tool for evaluation of function: the simple shoulder test. In: Matsen FA, Fu FH, Hawkins RJ (Hrsg) The shoulder: a balance of mobility and stability. The American Academy of Orthopedic Surgeons, Rosemont, S501-559

31. Macefield RC, Jacobs M, Korfage IJ et al (2014) Developing core outcomes sets: methods for identifying and including patient-reported outcomes (PROs). Trials 15:49

32. Magosch P (2017) In: Habermeyer P et al (Hrsg) Schulterchirurgie. Elsevier, München, S820-853

33. Marks $M$, Rickenbacher $D$, Audigé L, Glanzmann MC (2020) Patient-Rated Tennis Elbow Evaluation (PRTEE).Z Orthop Unfall. https://doi.org/10.1055/ a-1107-3313

34. Marquardt J, Schottker-Koniger T, Schafer A (2016) Validation of the German version of the Oxford Elbow Score : a cross-sectional study. Orthopade 45:695-700

35. Marquardt J, Tampin B, Schöttker-Königer T et al (2015) Die Übersetzung des „Oxford Elbow Score" (OES) in die deutsche Version der "Oxford Ellenbogen Bewertung" (OEB). Physioscience 11:150-156

36. Morrey BF, Adams RA (1992) Semiconstrained arthroplasty for the treatment of rheumatoid arthritis of the elbow. J Bone Joint Surg Am 74:479-490

37. Mosher ZA, Ewing MA, Collins CS, Young PG Brabston EW, Momaya AM, Tashjian RZ, Ponce BA (2020) Usage Trends of Patient-reported Outcome
Measures in Shoulder Literature. J Am Acad Orthop Surg 28(17):e774-e781. https://doi.org/10.5435/ JAAOS-D-19-00455

38. Nelson EC, Eftimovska E, Lind C et al (2015) Patient reported outcome measures in practice. BMJ 350:g7818

39. O'connor CM, Ring D (2019) Correlation of single assessment numeric evaluation (SANE) with other patient reported outcome measures (PROms). Arch Bone Jt Surg 7:303-306

40. Overend TJ, Wuori-Fearn JL, Kramer JF et al (1999) Reliability of a patient-rated forearm evaluation questionnaire for patients with lateral epicondylitis. JHand Ther 12:31-37

41. Riedel K, Beaton DE (2013) Update on the state of outcome measurement in total elbow arthroplasty research: identifying a need for consensus. J Bone Joint Surg Am 95:e97 1-8

42. Rotenstein LS, Huckman RS, Wagle NW (2017) Making patients and doctors happier-the potential of patient-reported outcomes. N Engl J Med 377:1309-1312

43. Rowe CR (1988) Evaluation of the shoulder. In The shoulder. Churchill Livingstone, New York, S631-637

44. Rowe CR, Patel D, Southmayd WW (1978) The Bankart procedure: a long-term end-result study. JBone Joint Surg Am 60:1-16

45. Rowe CR, Zarins B (1982) Chronic unreduced dislocations of the shoulder. J Bone Joint Surg Am 64:494-505

46. Rowe CR, Zarins B (1981) Recurrent transient subluxation of the shoulder. J Bone Joint Surg Am 63:863-872

47. Schneider MM, Nietschke R, Burkhart KJ, Hollinger B, Marquardt J, Schöttker-Königer T, Morrison F, Zimmerer A, Papen A (2020) Translation of the Mayo Elbow Performance Score (MEPS) into German (MEPS-G).ZOrthopUnfall 158(5):455-461.https:// doi.org/10.1055/a-0974-3836

48. Williamson PR, Altman DG, Bagley H et al (2017) The COMET handbook: version 1.0. Trials 18:280

49. Wright RW, Baumgarten KM (2010) Shoulder outcomes measures. J Am Acad Orthop Surg 18:436-444 